\title{
ÚNG DỤNG TOÁN THỔNG KÊ ĐỂ ĐÁNH GIÁ ĐỘ ỐN ĐỊNH MỐC CƠ SỞ ĐO LÚN CÔNG TRÌNH TỬ KẾT QUẢ ĐO NHIỀU CHU KỲ
}

\author{
ThS. TÓNG TH!̣ HẠNH ${ }^{(1)}$, ThS. BÙI TH!̣ KIÊN TRINH(2) \\ (1) Học viện Kỹ thuật Quân sự \\ (2) Trường Đại học Thuỷ lợi
}

\section{Tóm tắt:}

Trong công tác quan trắc lún công trình, sự ổn định của các mốc khống chế cơ sở quyết định độ chính xác của kết quả quan trắc. Dựa trên cơ sở của bài toán kiểm định thống kê, bài báo nhằm mục tiêu xác định tiêu chuẩn hợp lý để đánh giá độ ổn định của các mốc cơ sở phục vụ đo lún công trình từ kết quả quan trắc nhiều chu kỳ.

\section{Mở đầu}

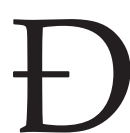
ể quan trắc chuyển dịch biến dạng các công trình công nghiệp hay dân dụng cần xây dựng hệ thống mốc đo đạc. Hệ thống này phân thành hai cấp: mốc khống chế cơ sở là các mốc chuẩn được bố trí ở khu vực ổn định và cấp mốc quan trắc gắn trên thân công trình, tại những vị trí đặc trưng dễ phát hiện chuyển dịch nhất.

Bản chất của công tác quan trắc lún công trình là đo chênh cao từ các mốc chuẩn đến các mốc quan trắc, từ đó tính được độ cao các mốc quan trắc theo phương pháp bình sai chặt chẽ. Thông qua so sánh độ cao của các mốc quan trắc ở các chu kỳ đo khác nhau sẽ xác định được độ lún của công trình trong quãng thời gian đó. Như vậy, muốn xác định chính xác độ lún của công trình thì các mốc chuẩn phải ổn định, việc đánh giá độ ổn định của các mốc này có ý nghĩa quyết định chất lượng của việc tính độ lún, tính các tham số lún và dự báo lún công trình, đồng thời cần thường xuyên được nghiên cứu và hoàn thiện.

Có rất nhiều phương pháp đánh giá độ ổn định mốc khống chế cơ sở (mốc chuẩn), nhưng các phương pháp đó chủ yếu dùng để đánh giá độ ổn định các mốc chuẩn lưới cơ sở từ kết quả đo hai chu kỳ. Một số rất ít các phương pháp dùng để đánh giá độ ổn định các mốc chuẩn từ kết quả đo nhiều chu kỳ như phương pháp phân tích tương quan, phương pháp dùng luật phân bố D-Simon .v.v... Tuy nhiên cơ sở khoa học và khả năng ứng dụng thực tiễn của các phương pháp là vấn đề cần phải xem xét và phân tích từ nhiều góc độ khác nhau. Trong bài báo này, chúng tôi sẽ phân tích cơ sở khoa học và khả năng ứng dụng của các phương pháp, từ đó hoàn thiện phương pháp đánh giá độ ổn định của mốc cơ sở đo lún từ kết quả đo nhiều chu kỳ dựa trên cơ sở của bài toán kiểm định thống kê.

2. Tổng quan về các phương pháp đánh giá độ ổn định mốc cơ sở từ kết quả đo nhiều chu kỳ

Các phương pháp đánh giá độ ổn định mốc cơ sở đo lún từ kết quả đo của nhiều chu kỳ hầu hết đều dựa trên cơ sở của bài toán kiểm định thống kê nhưng cách lựa chọn tiêu chuẩn và quy tắc kiểm định của mỗi phương pháp là khác nhau. Sau đây là nội dung cơ bản của một số phương pháp thông dụng.

\subsection{Phương pháp phân tích tương quan}

Giả thiết lưới cơ sở được đo k chu kỳ với 
$\mathrm{n}$ trị đo chênh cao là $\mathrm{h}_{\mathrm{iii}}$. Ký hiệu các chênh cao sau bình sai là $h_{\mathrm{ij}}^{\prime}$ và sai số trung phương tương ứng là

$$
\sigma_{h_{i j}^{\prime}} \text {. }
$$

Trong phương pháp phân tích tương quan, giả thiết thống kê $\mathrm{H}_{0}$ được tạo:

$$
\mathrm{H}_{0}: \quad \mathrm{h}_{\mathrm{i} 1}^{\prime}=\mathrm{h}_{\mathrm{i} 2}^{\prime}=\ldots=\mathrm{h}_{\mathrm{ik}}^{\prime}
$$

tức là chênh cao $\mathrm{h}_{\mathrm{ij}}^{\prime}$ cố định trong $\mathrm{k}$ chu kỳ đo.

Quy tắc để kiểm định giả thiết thống kê (1) là đại lượng thống kê:

$$
F=\frac{\sigma_{\bar{h}_{i}}^{2}}{\sigma_{\mathrm{h}_{\mathrm{ij}}^{\prime}}^{2}}
$$

Trong đó: $\sigma_{\bar{h}_{i}}^{2}$ là bình phương sai số trung phương cua trị chênh cao trung bình từ $\mathrm{k}$ chu kỳ đo được tính theo công thức:

$$
\sigma_{\bar{h}_{\mathrm{i}}}^{2}=\frac{\sum_{\mathrm{j}=1}^{\mathrm{k}}\left(\mathrm{h}_{\mathrm{ij}}^{\prime}-\overline{\mathrm{h}}_{\mathrm{i}}\right)^{2}}{\mathrm{k}(\mathrm{k}-1)}
$$

Đại lượng thống kê (3) sẽ có luật phân bố Fish-Snedec với số bậc tự do $\{k(k-1), r\}$. Tính được trị thực tế $\mathrm{f}_{\mathrm{p}}$ theo công thức (3) và kiểm tra giả thiết (1) theo nguyên tắc:

a. Nếu $\mathrm{f}_{\mathrm{p}} \leq \mathrm{f}_{\alpha,\{k(\mathrm{k}-1), \mathrm{r}\}}$ ta chấp nhận giả thiết (1), ngnıa ıa tr!̣ bınh sai chênh cao $\mathrm{h}_{\mathrm{ij}}^{\prime}$ cố định trong $\mathrm{k}$ chu kỳ đo.

b. Ngược lại, nếu $\mathrm{f}_{\mathrm{p}}>\mathrm{f}_{\alpha,\{\mathrm{k}(\mathrm{k}-1), \mathrm{r}\}}$ thì giả thiết (1) bị phủ định, nghĩa là trị binh sai chênh cao $\mathrm{h}_{\mathrm{ij}}^{\prime}$ không cố định trong $\mathrm{k}$ chu kỳ đo.

Từ đó rút ra kết luận:

- Nếu tất cả các chênh cao trong lưới đều có kết luận là cố định trong $\mathrm{k}$ chu kỳ đo thì không phải tiến hành xác định mốc chuẩn ổn định. Trong trường hợp này có thể là tất cả các mốc chuẩn đều ổn định hoặc tất cả các mốc lún đều.

- Trường hợp có ít nhất một chênh cao có kết luận không cố định, lúc này phải xác định mốc chuẩn ổn định và mốc chuẩn không ổn định theo cách phân tích dựa vào cấu trúc lưới hoặc dựa vào giá trị của các hệ số tương quan điều kiện.

\subsection{Phương pháp dùng luật phân bố D-Simon}

Phương pháp dùng luật phân bố DSimon được trình bày trong tài liệu tham khảo [2]. Phương pháp này được thực hiện với từng mốc chuẩn của lưới cơ sở. Với lưới cơ sở đo lún gồm $p$ mốc chuẩn được đo k chu kỳ, nội dung của phương pháp có thể tóm tắt qua 3 bước như sau:

Bước 1: Từ độ chính xác quan trắc lún tổng hợp $\sigma_{\Delta_{\text {yéu }}}$ sẽ tính được độ chính xác đo lún yêu cau của cấp lưới cơ sở theo công thức:

$$
\sigma_{\Delta_{\mathrm{cs}}}=\frac{\sigma_{\Delta_{\text {yé́u }}}}{\sqrt{1+\mathrm{K}^{2}}}
$$

Tiến hành bình sai lưới theo phương pháp bình sai lưới tự do, có thể bình sai theo chênh cao đo hoặc theo hiệu chênh cao đo. Nếu bình sai theo chênh cao đo cần lưu ý là khi bình sai các chu kỳ sau ta nhận trị gần đúng của các ẩn số (độ cao mốc chuẩn) bằng trị bình sai độ cao các mốc tương ứng ở chu kỳ bình sai trước.

\section{Bước 2:}

a. Xác định độ lún $\Delta_{\mathrm{ij}}$ và sai số trung phương độ lún $\sigma_{\Delta_{\mathrm{ij}}}$ với $(\mathrm{i}=1 \div \mathrm{p})$ và

$$
(\mathrm{j}=1 \div \mathrm{k})
$$

Việc xác định hai đại lượng này sẽ phụ thuộc vào đối tượng trị đo được sử dụng trong bài toán bình sai lưới:

- Nếu bình sai lưới theo dãy hiệu chênh cao đo thì sẽ nhận được ngay độ lún $\Delta_{\mathrm{ij}}$ và sai số trung phương độ lún $\sigma_{\Delta_{\mathrm{ij}}}$.

- Nếu bình sai theo dãy chênh cao đo, dựa vào trị bình sai độ cao $\mathrm{H}_{\mathrm{ij}}$, trọng số đảo tương ứng $\mathrm{Q}_{\mathrm{H}_{\mathrm{ij}}}$ và sai số trung phương 
trọng số đơn vị $\sigma_{0 \mathrm{j}}$ ta xác định được:

+ Độ lún: $\quad \Delta_{\mathrm{ij}}=\mathrm{H}_{\mathrm{ij}}-\mathrm{H}_{\mathrm{i}(\mathrm{j}-1)}$

+ Sai số trung phương độ lún theo công thức:

$$
\sigma_{\Delta_{\mathrm{ij}}}=\bar{\sigma}_{0_{\mathrm{ij}}} \sqrt{\mathrm{Q}_{\Delta_{\mathrm{ij}}}}=\bar{\sigma}_{0_{\mathrm{ij}}} \sqrt{\mathrm{Q}_{\mathrm{H}_{\mathrm{ij}}}+\mathrm{Q}_{\mathrm{H}_{\mathrm{i}(\mathrm{j}-1)}}}
$$

Trong đó:

$$
\bar{\sigma}_{0_{i j}}=\frac{r_{(j-1)} \sigma_{0(j-1)}^{2}+r_{j} \sigma_{0 j}^{2}}{r_{(j-1)}+r_{j}}
$$

b. Tạo dãy độ lún cùng độ chính xác.

Từ dãy độ lún $\Delta_{\mathrm{ij}}$ của mốc chuẩn (i) khác độ chính xác chúng ta phải cân bằng chúng bằng cách tạo dãy độ lún tương đương:

$$
\Delta_{\mathrm{ij}}^{\prime}=\Delta_{\mathrm{ij}} \sqrt{\mathrm{P}_{\Delta_{\mathrm{ij}}}}
$$

Trong đó: $\mathrm{P}_{\Delta_{\mathrm{ij}}}$ à trọng số của độ lún $\Delta_{\mathrm{ij}}$ và được xác định theo một trong các cách sau:

- Nếu yêu cầu xác định độ lún các mốc cơ sở là $\sigma_{\Delta}$ thì chọn trọng số theo công thức:

$$
\mathrm{P}_{\Delta_{\mathrm{ij}}}=\frac{\sigma_{\Lambda}^{2}}{\sigma_{\Delta_{\mathrm{ij}}}^{2}}
$$

- Khi giãn cách thời gian giữa các chu kỳ chênh lệch lớn thì trọng số $\mathrm{P}_{\Delta_{\mathrm{ij}}}$ có thể chọn
theo công thức:

$$
\mathrm{P}_{\Delta_{\mathrm{ij}}}=\frac{\mathrm{T}_{0}}{\mathrm{~T}_{\mathrm{i}}} \frac{\sigma_{\Delta}^{2}}{\sigma_{\Delta_{\mathrm{ij}}}^{2}}
$$

Trong đó: $T_{0}$ chọn phù thuộc vào thời gian giữa các chu kỳ dùng để tính các độ lún.

- Dựa vào kết quả bình sai ta cũng có thể xác định trọng số của độ lún theo công thức:

$$
\mathrm{P}_{\Delta_{\mathrm{ij}}}=\frac{1}{\mathrm{Q}_{\Delta_{\mathrm{ij}}}}
$$

Bước 3: Đánh giá độ ổn định của các mốc chuẩn.

Để đánh giá độ ổn định của mốc chuẩn (i) trong dãy $\mathrm{k}$ chu kỳ đo ta tạo giả thiết:

$\mathrm{H}_{0}: \mathrm{E}\left(\Delta_{\mathrm{i} 1}\right)=\mathrm{E}\left(\Delta_{\mathrm{i} 2}\right)=\ldots=\mathrm{E}\left(\Delta_{\mathrm{i}(\mathrm{k}-1)}\right)=0$

Dùng đại lượng thống kê:

$$
\mathrm{D}=\frac{\mathrm{R}_{\max }}{\sigma_{\Delta}}=\frac{\Delta_{\max }-\Delta_{\min }}{\sigma_{\Delta}}
$$

làm quy tắc để kiểm định giả thiết (12). Đại lượng thống kê (13) sẽ có luật phân bố DSimon với số bậc tự do (k-1).

Trong quy tắc (13), để tính trị thực tế của quy tắc chúng tôi đã nhận trị cực đại $\Delta_{\max }=\left|\Delta^{\prime}\right|_{\max }$ và trị cực tiểu $\Delta_{\text {min }}=0$. Chọn mức xác suất $\alpha$ và tra bảng $D$-Simon ta tìm được trị giới hạn $\mathrm{d}_{\alpha,(\mathrm{k}-1)}$, sau đó so sánh với trị thực tế $\mathrm{d}_{\mathrm{p}}$ của quy tắc (13) và rút ra kết luận:

+ Nếu $\mathrm{d}_{\mathrm{p}} \leq \mathrm{d}_{\alpha,(\mathrm{k}-1)}$ : chấp nhận giả thiết (12), nghĩa là mốc chuẩn (i) ổn định trong $\mathrm{k}$ chu kỳ đo.

+ Ngược lại, nếu $d_{p}>d_{\alpha,(k-1)}$ ta nói mốc chuẩn (i) không ổn định trong $\mathrm{k}$ chu kỳ đo, nghĩa là có ít nhất một chu kỳ đo có mốc chuẩn (i) không ổn định.

\section{Lựa chọn phương pháp hợp lý để đánh giá độ ổn định mốc đo lún nhiều chu kỳ}

Để lựa chọn một phương pháp hợp lý nhằm đánh giá độ ổn định các mốc đo lún nhiều chu kỳ chúng ta nên dựa vào các mục tiêu sau:

Mục tiêu 1: Phương pháp phải được xây dựng trên cơ sở toán học chặt chẽ và phải phù hợp với tính thực tế độ lún các mốc.

Mục tiêu 2: Quy trình tính toán của phương pháp đơn giản, có khối lượng tính 
toán ít nhất, có tính phổ cập cao. Đồng thời phương pháp phải có khả năng tự động hóa quá trình tính toán.

Dựa vào hai mục tiêu trên, chúng tôi sẽ phân tích ưu nhược điểm của từng phương pháp từ đó lựa chọn phương pháp hợp lý nhất.

\section{- Phương pháp phân tích tương quan:}

Xét về mặt cơ sở lý thuyết thì phương pháp này có cơ sở toán học chặt chẽ vì nếu mốc cơ sở không bị lún thì chênh cao trong các chu kỳ tương ứng sẽ như nhau.

Tuy nhiên, phương pháp này có khối lượng tính toán lớn, đặc biệt khi lưới cơ sở có số điểm mốc nhiều. Đồng thời khả năng tự động hóa của phương pháp không cao vì trong trường hợp có ít nhất một chênh cao không ổn định thì việc xác định mốc không ổn định sẽ rất phức tạp vì sẽ phải phân tích dựa vào cấu trúc lưới hoặc giá trị của các hệ số tương quan.

\section{Simon:}

- Phương pháp dùng luật phân bố $D$ -

Phương pháp dùng luật phân bố DSimon cũng dựa trên cơ sở bài toán kiểm định thống kê có giả thiết thống kê và quy tắc kiểm định hợp lý. Tuy nhiên, trọng số của độ lún các mốc cơ sở có nhiều công thức sẽ gây khó khăn khi lựa chọn công thức tính. Đồng thời phương pháp chưa xác định được chu kỳ đo có mốc chuẩn không ổn định mặc dù đây là một việc cần thiết.

Phương pháp có ưu điểm là có quy trình tính toán rõ ràng và có tính quy luật, có khả năng tự động hóa quá trình tính toán.

Trên cơ sở những phân tích trên, chúng tôi đã kế thừa những ưu điểm và hoàn thiện các tồn tại của phương pháp dùng luật phân bố $\mathrm{D}$-Simon nhằm đánh giá độ ổn định mốc chuẩn trong đo lún công trình, tóm tắt như sau:

3.1. Chọn công thức tính trọng số độ lún hợp lý

Trong ba cách chọn trọng số theo (9), (10), (11) đã nêu, có thể thấy công thức (11) phù hợp nhất với lưới khống chế quan trắc lún vì trọng số độ lún tỷ lệ nghịch với trọng số đảo độ lún. Mặt khác, khi đưa trọng số đảo vào tính độ lún thì sẽ thể hiện được sự tác động của cấu hình lưới ở chu kỳ đo tương ứng đến độ lún.

\subsection{Xác định chu kỳ đo có mốc chuẩn không ổn định}

Để xác định chu kỳ đo có mốc chuẩn không ổn định chúng ta tiến hành như sau:

- Khi nhận trị cực đại $\Delta_{\max }=\left|\Delta^{\prime}\right|_{\max }$ và trị cực tiểu $\Delta_{\min }=0$ thì quy tắc (13) có dạng:

$$
\mathrm{D}=\frac{\left|\Delta^{\prime}\right|_{\max }}{\sigma_{\Delta}}
$$

- Đại lượng thống kê (14) có luật phân bố D-Simon. Tính trị thực tế $\mathrm{d}_{\mathrm{p}}$ của quy tắc theo (14) và so sánh với trị tới hạn $\mathrm{d}_{\alpha,(\mathrm{k}-1)}$ tra từ bảng tra của luật phân bố D-Sımon:

+ Nếu $\mathrm{d}_{\mathrm{p}} \leq \mathrm{d}_{\alpha,(\mathrm{k}-1)}$ thì giả thiết (12) được chấp nhận, tức là mốc chuẩn (i) ổn định trong tất cả các chu kỳ đo và kết thúc bài toán đánh giá độ ổn định.

+ Nếu $d_{p}>d_{\alpha,(k-1)}$ thì giả thiết (12) bị bác bỏ, tức là mốc chuẩn (i) không ổn định tại chu kỳ có độ lún cực đại, giả sử tại chu kỳ (j) là $\left|\Delta^{\prime}\right|_{\max (j)}$. Để kiểm tra xem liệu rằng còn chu kỳ nào có mốc chuẩn (i) không ổn định nữa hay không thì chúng ta tiến hành loại bỏ $\left|\Delta^{\prime}\right|_{\max (\mathrm{j})}$ ở chu kỳ (j), sau đó kiểm nghiệm đối với $\left|\Delta^{\prime}\right|_{\max }$ trong dãy các chu kỳ còn lại, nếu giả thiết gốc bị bác bỏ tức là còn có chu kỳ đo có mốc không ổn định thì tiếp tục kiểm nghiệm cho đến khi tìm được tất cả các chu kỳ có mốc chuẩn không ổn định, 
các chu kỳ còn lại là các chu kỳ đo có mốc chuẩn ổn định.

\section{Tính toán thực nghiệm}

Để minh chứng cho các phân tích trên, chúng tôi tiến hành thực nghiệm kiểm tra độ ổn định của 4 mốc $R_{1}, R_{2}, R_{3}, R_{4}$ của lưới cơ sở ở hình 1. Lưới được xây dựng nhằm đo lún công trình với độ chính xác $\sigma_{\Delta_{\text {уби }}}= \pm 1 \mathrm{~mm}$ nên $\sigma_{\Delta_{\mathrm{cs}}}=0.32 \mathrm{~mm}$.

Lưới được đo 9 chu kỳ, số liệu đo ghi ở bảng 1. (Xem bảng 1)

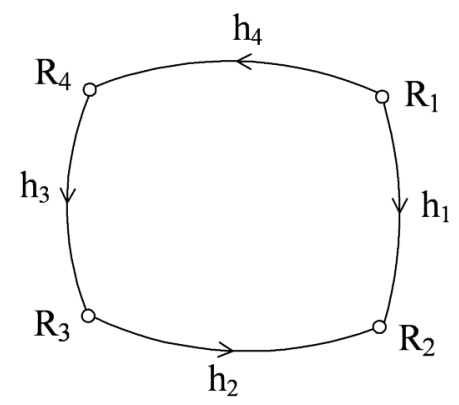

Hình 1: Sơ đồ lưới thực nghiệm
Sau khi bình sai thu được các kết quả trong bảng 2: (Xem bảng 2, 3)

Từ kết quả bình sai, tiến hành đánh giá độ ổn định các mốc chuẩn theo hai phương pháp gồm: phương pháp phân tích tương quan và phương pháp dùng luật phân bố $D$ Simon đã hoàn thiện. Cụ thể như sau:

a. Đánh giá độ ổn định các mốc chuẩn theo phương pháp phân tích tương quan (Bảng 4).

Trị giới hạn:

$$
\mathrm{f}_{\text {g.hạn }}=\mathrm{f}_{\alpha, \mathrm{k}(\mathrm{k}-1), 1}=\mathrm{f}_{0.05,9(9-1), 1}=3.98
$$

So sánh các giá trị thực tế $\mathrm{f}_{\mathrm{p} 1}, \mathrm{f}_{\mathrm{p} 2}, \mathrm{f}_{\mathrm{p} 3}, \mathrm{f}_{\mathrm{p} 4}$ với trị giới hạn $\mathrm{f}_{\mathrm{g} . h a ̣ n}$, chúng tôi rút ra kết luận sau:

- Chênh cao $\mathrm{h}_{1}$ là không cố định trong 9 chu kỳ đo.

- Chênh cao $\mathrm{h}_{2}$ là không cố định trong 9 chu kỳ đo.

Bảng 1: Số liệu thực nghiệm

\begin{tabular}{|c|c|c|c|c|c|c|c|c|}
\hline Chu kỳ & $\begin{array}{c}\mathrm{h}_{1} \\
(\mathrm{~mm})\end{array}$ & $\begin{array}{c}\mathrm{n}_{1} \\
(\text { Tram })\end{array}$ & $\begin{array}{c}\mathrm{h}_{2} \\
(\mathrm{~mm})\end{array}$ & $\begin{array}{c}\mathrm{n}_{2} \\
(\text { Trạm })\end{array}$ & $\begin{array}{c}\mathrm{h}_{3} \\
(\mathrm{~mm})\end{array}$ & $\begin{array}{c}\mathrm{n}_{3} \\
(\text { Trạm })\end{array}$ & $\begin{array}{c}\mathrm{h}_{4} \\
(\mathrm{~mm})\end{array}$ & $\begin{array}{c}\mathrm{n}_{4} \\
(\text { Trạm })\end{array}$ \\
\hline 1 & 979.52 & 4 & 484.28 & 3 & 398.84 & 4 & 96.67 & 5 \\
\hline 2 & 980.31 & 4 & 483.92 & 3 & 398.49 & 4 & 97.58 & 5 \\
\hline 3 & 979.97 & 3 & 483.24 & 5 & 398.33 & 5 & 97.60 & 5 \\
\hline 4 & 978.71 & 3 & 481.53 & 5 & 398.84 & 5 & 98.13 & 6 \\
\hline 5 & 977.31 & 3 & 481.16 & 4 & 399.09 & 4 & 96.75 & 4 \\
\hline 6 & 976.77 & 3 & 480.68 & 4 & 398.96 & 5 & 96.22 & 4 \\
\hline 7 & 976.52 & 5 & 480.12 & 4 & 399.18 & 3 & 96.92 & 5 \\
\hline 8 & 978.15 & 3 & 481.83 & 3 & 398.79 & 5 & 97.11 & 5 \\
\hline 9 & 974.72 & 4 & 479.24 & 4 & .98 .96 & 3 & 96.14 & 4 \\
\hline
\end{tabular}


Bảng 2: Độ cao các mốc và trọng số đảo độ cao các mốc sau bình sai

\begin{tabular}{|c|c|c|c|c|c|c|c|c|}
\hline Chu kỳ & $\begin{array}{c}\mathrm{H}_{\mathrm{R} 1} \\
(\mathrm{~m})\end{array}$ & $\begin{array}{c}\mathrm{H}_{\mathrm{R} 2} \\
(\mathrm{~m})\end{array}$ & $\begin{array}{c}\mathrm{H}_{\mathrm{R} 3} \\
(\mathrm{~m})\end{array}$ & $\begin{array}{c}\mathrm{H}_{\mathrm{R} 4} \\
(\mathrm{~m})\end{array}$ & $\mathrm{Q}_{\mathrm{H}_{\mathrm{R} 1}}$ & $\mathrm{Q}_{\mathrm{H}_{\mathrm{R} 2}}$ & $\mathrm{Q}_{\mathrm{H}_{\mathrm{R} 3}}$ & $\mathrm{Q}_{\mathrm{H}_{\mathrm{R}}}$ \\
\hline 1 & 9.99997 & 10.97956 & 10.49533 & 10.09656 & 1.36 & 1.11 & 1.11 & 1.36 \\
\hline 2 & 9.99932 & 10.97955 & 10.49557 & 10.09700 & 1.36 & 1.11 & 1.11 & 1.36 \\
\hline 3 & 9.99935 & 10.97919 & 10.49572 & 10.09717 & 1.25 & 1.25 & 1.53 & 1.53 \\
\hline 4 & 9.99940 & 10.97806 & 10.49645 & 10.09753 & 1.36 & 1.28 & 1.54 & 1.67 \\
\hline 5 & 10.00034 & 10.97758 & 10.49634 & 10.09717 & 1.10 & 1.10 & 1.23 & 1.23 \\
\hline 6 & 10.00067 & 10.97727 & 10.49636 & 10.09712 & 1.11 & 1.11 & 1.36 & 1.36 \\
\hline 7 & 10.00044 & 10.97687 & 10.49668 & 10.09745 & 1.51 & 1.37 & 1.13 & 1.22 \\
\hline 8 & 9.99999 & 10.97806 & 10.69615 & 10.09723 & 1.19 & 1.00 & 1.19 & 1.50 \\
\hline 9 & 10.00132 & 10.97594 & 10.49660 & 10.09756 & 1.23 & 1.23 & 1.10 & 1.10 \\
\hline
\end{tabular}

Bảng 3: Chênh cao sau bình sai, chênh cao sau bình sai trung bình và sai số trung phương xác định chênh cao sau bình sai

\begin{tabular}{|c|c|c|c|c|c|c|c|c|}
\hline Chu kỳ & $\begin{array}{c}\mathrm{h}_{1} \\
(\mathrm{~mm})\end{array}$ & $\begin{array}{c}\mathrm{h}_{2} \\
(\mathrm{~mm})\end{array}$ & $\begin{array}{c}\mathrm{h}_{3} \\
(\mathrm{~mm})\end{array}$ & $\begin{array}{c}\mathrm{h}_{4} \\
(\mathrm{~mm})\end{array}$ & $\begin{array}{c}\mathrm{m}_{\mathrm{h}_{1}} \\
(\mathrm{~mm})\end{array}$ & $\begin{array}{c}\mathrm{m}_{\mathrm{h}_{2}} \\
(\mathrm{~mm})\end{array}$ & $\begin{array}{c}\mathrm{m}_{\mathrm{h}_{3}} \\
(\mathrm{~mm})\end{array}$ & $\begin{array}{c}\mathrm{m}_{\mathrm{h}_{4}} \\
(\mathrm{~mm})\end{array}$ \\
\hline 1 & 979.59 & 484.23 & 398.77 & 96.59 & 0.12 & 0.11 & 0.12 & 0.13 \\
\hline 2 & 980.23 & 483.98 & 398.57 & 97.68 & 0.14 & 0.12 & 0.14 & 0.15 \\
\hline 3 & 979.84 & 483.46 & 398.55 & 97.82 & 0.30 & 0.36 & 0.36 & 0.36 \\
\hline 4 & 978.66 & 481.61 & 398.92 & 98.23 & 0.11 & 0.14 & 0.14 & 0.14 \\
\hline 5 & 977.25 & 481.24 & 399.17 & 96.83 & 0.12 & 0.14 & 0.14 & 0.14 \\
\hline 6 & 976.60 & 480.91 & 399.24 & 96.45 & 0.36 & 0.39 & 0.42 & 0.39 \\
\hline 7 & 976.43 & 480.19 & 399.23 & 97.01 & 0.14 & 0.13 & 0.11 & 0.14 \\
\hline 8 & 978.07 & 481.91 & 398.92 & 97.24 & 0.16 & 0.16 & 0.19 & 0.19 \\
\hline \hline 9 & 974.62 & 479.34 & 399.04 & 96.24 & 0.17 & 0.17 & 0.15 & 0.17 \\
\hline$\overline{\mathrm{h}}_{\mathrm{i}}$ & 977.92 & 481.87 & 398.94 & 97.12 & & & & \\
\cline { 1 - 4 } & & & &
\end{tabular}


Bảng 4: Kiểm định sự ổn định của chênh cao $h_{i}$ trong $k$ chu kỳ đo

\begin{tabular}{|c|c|c|c|c|c|c|c|c|}
\hline \multirow[b]{2}{*}{$\begin{array}{l}\text { Chu } \\
\text { kỳ }\end{array}$} & \multicolumn{2}{|c|}{ Kiểm định $\mathrm{h}_{1}$} & \multicolumn{2}{|c|}{ Kiểm định $\mathrm{h}_{2}$} & \multicolumn{2}{|c|}{ Kiểm định $\mathrm{h}_{3}$} & \multicolumn{2}{|c|}{ Kiểm định $\mathrm{h}_{4}$} \\
\hline & $\left(\mathrm{h}_{\mathrm{ij}}^{\prime}-\overline{\mathrm{h}}_{1}\right)^{2}$ & $f_{p 1}=\frac{\sigma_{\bar{h}_{1}^{\prime}}^{2}}{\sigma_{h_{1 j}^{\prime}}^{2}}$ & $\left(\mathrm{~h}_{2 \mathrm{j}}^{\prime}-\overline{\mathrm{h}}_{2}\right)$ & $f_{p 2}=\frac{\sigma_{\bar{h}_{2}^{\prime}}^{2}}{\sigma_{h_{2 i}^{\prime}}^{2}}$ & $\left(\mathrm{~h}_{3 \mathrm{j}}^{\prime}-\overline{\mathrm{h}}_{3}\right)^{2}$ & $f_{p 3}=\frac{\sigma_{h_{3}^{\prime}}^{2}}{\sigma_{h_{3 j}^{\prime}}^{2}}$ & $\left(\mathrm{~h}_{4 \mathrm{j}}^{\prime}-\overline{\mathrm{h}}_{4}\right)^{2}$ & $f_{p 4}=\frac{\sigma_{\bar{h}_{4}^{\prime}}^{2}}{\sigma_{\mathrm{h}_{4 j}^{\prime}}^{2}}$ \\
\hline 1 & 2.781 & 30.288 & 5.567 & 29.174 & 0.028 & 0.667 & 0.286 & 3.384 \\
\hline 2 & 5.336 & 21.563 & 4.452 & 20.769 & 0.137 & 0.100 & 0.314 & 2.409 \\
\hline 3 & 3.674 & 4.657 & 2.535 & 2.523 & 0.150 & 0.687 & 0.493 & 0.413 \\
\hline 4 & 0.549 & 32.400 & 0.067 & 17.387 & 0.000 & 0.681 & 1.227 & 2.552 \\
\hline 5 & 0.452 & 26.925 & 0.394 & 17.241 & 0.054 & 0.072 & 0.083 & 2.820 \\
\hline 6 & 1.744 & 3.282 & 0.926 & 2.087 & 0.093 & 0.979 & 0.452 & 0.341 \\
\hline 7 & 2.215 & 22.157 & 2.820 & 20.008 & 0.086 & 0.338 & 0.012 & 2.836 \\
\hline 8 & 0.023 & 15.406 & 0.002 & 12.057 & 0.000 & 0.390 & 0.015 & 1.398 \\
\hline 9 & 10.899 & 17.352 & 6.394 & 10.978 & 0.009 & 0.390 & 0.772 & 2.479 \\
\hline & $\sigma_{\mathrm{h}_{1}^{\prime}}^{2}=\frac{\sum_{\mathrm{j}=1}^{9}\left(\mathrm{~h}_{1}^{\prime}\right.}{9(\mathrm{~S}}$ & $\frac{\left.5_{1}\right)^{2}}{5}=$ & $\sigma_{\hat{h}_{2}^{\prime}}^{2}=\frac{\sum_{j=1}^{9}\left(h_{2}^{\prime}\right.}{9(}$ & $\frac{\left.\overline{\mathrm{h}}_{2}\right)^{2}}{\text { 1) }}$ & $\sigma_{\mathrm{h}_{h^{\prime}}}^{2}=\frac{\sum_{j=1}^{9}\left(h_{3 j}^{\prime}\right.}{9(9}$ & $\frac{i_{3}}{2}$ & $\sigma_{\mathrm{h}_{4}^{\prime}}^{2}=\frac{\sum_{\mathrm{j}=1}^{9}(1}{9}$ & \\
\hline
\end{tabular}

- Chênh cao $h_{3}$ là cố định trong 9 chu kỳ đo.

- Chênh cao $\mathrm{h}_{4}$ là cố định trong 9 chu kỳ đo.

Kết hợp với việc phân tích sơ đồ lưới chúng ta thấy: mốc $R_{2}$ liên quan đến các chênh cao không cố định $h_{1}, h_{2}$ nên mốc $R_{2}$ là mốc không ổn định. Các mốc $R_{1}, R_{3}, R_{4}$ là mốc đầu và mốc cuối của các chênh cao cố định $\mathrm{h}_{3}, \mathrm{~h}_{4}$ nên là các mốc ổn định hoặc lún đều.

b. Đánh giá độ ổn định các mốc chuẩn theo phương pháp dùng luật phân bố $D$ simon.

Dựa vào độ cao và trọng số đảo độ cao các mốc sau bình sai chúng tôi tính được: (Xem bảng $5,6,7$ ) 
Bảng 5: Kết quả tính độ lún và trọng số đảo độ lún các mốc theo từng cặp chu kỳ liên tiếp

\begin{tabular}{|c|c|c|c|c|c|c|c|c|}
\hline $\begin{array}{c}\text { Căp chu } \\
\text { ky }\end{array}$ & $\begin{array}{c}\Delta_{\mathrm{R} 1} \\
(\mathrm{~mm})\end{array}$ & $\begin{array}{c}\Delta_{\mathrm{R} 2} \\
(\mathrm{~mm})\end{array}$ & $\begin{array}{c}\Delta_{\mathrm{R} 3} \\
(\mathrm{~mm})\end{array}$ & $\begin{array}{c}\Delta_{\mathrm{R} 4} \\
(\mathrm{~mm})\end{array}$ & $\mathrm{Q}_{\Delta_{\mathrm{R} 1}}$ & $\mathrm{Q}_{\Delta_{\mathrm{R} 2}}$ & $\mathrm{Q}_{\Delta_{\mathrm{R} 3}}$ & $\mathrm{Q}_{\Delta_{\mathrm{R} 4}}$ \\
\hline $1-2$ & -0.66 & -0.01 & 0.23 & 0.44 & 2.72 & 2.22 & 2.22 & 2.72 \\
\hline $2-3$ & 0.03 & -0.36 & 0.16 & 0.17 & 2.61 & 2.36 & 2.64 & 2.89 \\
\hline $3-4$ & 0.05 & -1.13 & 0.72 & 0.35 & 2.61 & 2.53 & 3.07 & 3.20 \\
\hline $4-5$ & 0.94 & -0.47 & -0.11 & -0.36 & 2.46 & 2.38 & 2.77 & 2.90 \\
\hline $5-6$ & 0.34 & -0.31 & 0.02 & -0.05 & 2.21 & 2.21 & 2.59 & 2.59 \\
\hline $6-7$ & -0.24 & -0.40 & 0.31 & 0.33 & 2.62 & 2.48 & 2.49 & 2.58 \\
\hline $7-8$ & -0.45 & 1.19 & -0.53 & -0.22 & 2.70 & 2.37 & 2.32 & 2.72 \\
\hline $8-9$ & 1.33 & -2.12 & 0.45 & 0.33 & 2.42 & 2.23 & 2.29 & 2.60 \\
\hline
\end{tabular}

Bảng 6: Kết quả tính trọng số độ lún và độ lún sau khi cân bằng trọng số

\begin{tabular}{|c|c|c|c|c|c|c|c|c|}
\hline $\begin{array}{c}\text { Căp chu } \\
\text { kỳ }\end{array}$ & $\mathrm{P}_{\Delta_{\mathrm{R}_{1}}}$ & $\mathrm{P}_{\Delta_{\mathrm{R}_{2}}}$ & $\mathrm{P}_{\Delta_{\mathrm{R}_{3}}}$ & $\mathrm{P}_{\Delta_{\mathrm{R}_{4}}}$ & $\begin{array}{c}\Delta_{\mathrm{R}_{1}}^{\prime} \\
(\mathrm{mm})\end{array}$ & $\begin{array}{c}\Delta_{\mathrm{R}_{2}}^{\prime} \\
(\mathrm{mm})\end{array}$ & $\begin{array}{c}\Delta_{\mathrm{R}_{3}}^{\prime} \\
(\mathrm{mm})\end{array}$ & $\begin{array}{c}\Delta_{\mathrm{R}_{4}}^{\prime} \\
(\mathrm{mm})\end{array}$ \\
\hline $1-2$ & 0.37 & 0.45 & 0.45 & 0.37 & -0.40 & -0.01 & 0.16 & 0.27 \\
\hline $2-3$ & 0.38 & 0.42 & 0.38 & 0.35 & 0.02 & -0.24 & 0.10 & 0.10 \\
\hline $3-4$ & 0.38 & 0.40 & 0.33 & 0.31 & 0.03 & -0.71 & 0.41 & 0.20 \\
\hline $4-5$ & 0.41 & 0.42 & 0.36 & 0.34 & 0.60 & -0.31 & -0.06 & -0.21 \\
\hline $5-6$ & 0.45 & 0.45 & 0.39 & 0.39 & 0.23 & -0.21 & 0.01 & -0.03 \\
\hline $6-7$ & 0.38 & 0.40 & 0.40 & 0.39 & -0.15 & -0.26 & 0.20 & 0.20 \\
\hline $7-8$ & 0.37 & 0.42 & 0.43 & 0.37 & -0.27 & 0.77 & -0.35 & -0.13 \\
\hline $8-9$ & 0.41 & 0.45 & 0.44 & 0.38 & 0.86 & -1.42 & 0.30 & 0.21 \\
\hline
\end{tabular}


Bảng 7: Kết quả đánh giá độ ổn định theo phương pháp D-Simon

\begin{tabular}{|c|c|c|c|c|}
\hline TT & Tên mốc & $\begin{array}{c}\text { Trị thực tế của quy } \\
\text { tắc } \mathrm{D}\end{array}$ & $\begin{array}{c}\text { Trị giới hăn của quy } \\
\text { tắc } \mathrm{D}\end{array}$ & Kết luận \\
\hline 1 & $\mathrm{R}_{1}$ & 2.68 & 4.29 & $\mathrm{R}_{1}$ ổn định \\
\hline 2 & $\mathrm{R}_{2}$ & 4.43 & 4.29 & $\begin{array}{c}\mathrm{R}_{2} \text { không ổn định tại chu } \\
\text { kỳ } 9\end{array}$ \\
\hline 3 & $\mathrm{R}_{3}$ & 1.29 & 4.29 & $\mathrm{R}_{3}$ ổn định \\
\hline 4 & $\mathrm{R}_{4}$ & 0.83 & 4.29 & $\mathrm{R}_{4}$ ổn định \\
\hline
\end{tabular}

\section{Kết luận}

Từ kết quả phân tích và tính toán thực nghiệm có thể thấy phương pháp sử dụng luật phân bố D-Simon đã hoàn thiện có quy luật tính toán chặt chẽ nhưng đơn giản, có thể tự động hóa quá trình tính toán. Đồng thời phương pháp sử dụng luật phân bố $\mathrm{D}$ simon đã hoàn thiện có độ tin cậy cao hơn phương pháp phân tích tương quan và không hạn chế số lượng mốc chuẩn trong lưới. Đặc biệt phương pháp này có thể áp dụng đối với cấp lưới quan trắc khi cần thiết. $O$

\section{Tài liệu tham khảo}

[1]. Trương Quang Hiếu (1996), Cơ sở toán học của lý thuyết sai số, Bài giảng cao học, Đại học Mỏ - Địa chất, Hà Nội.

[2]. Tống Thị Hạnh (2002), Nghiên cứu ứng dụng bài toán kiểm định thống kê để đánh giá độ ổn định mốc đo lún công trình, Luận văn Thạc sỹ Kỹ thuật, Đại học Mỏ - Địa chất, Hà Nội.

[3]. Phan Văn Hiến (1997), Quan trắc chuyển dịch và biến dạng công trình, Bài giảng cao học, Đại học Mỏ - Địa chất, Hà Nội.O

\section{Summary}

APPLY STATISTIC TO EVALUATE THE STABILITY SUBSIDENCE BENCHMARK FROM RESULTS MEASURED OF PERIODS

\section{MSc. Tong Thi Hanh, MSc. Bui Thi Kien Trinh}

In the process of monitoring subsidence, the stability of basic net stick determines the accuracy of the monitoring results. Based on statistical hypothesis testing, the paper aims at defining suitable standards to evaluate the stability of basis net stick for the settlement measurement of the project from monitoring results of periods. $O$

Ngày nhận bài 18/3/2013. 